\title{
A Measurable Stability Theorem for Holomorphic Foliations Transverse to Fibrations
}

\author{
Bruno Scardua \\ Instituto de Matematica, Universidade Federal do Rio de Janeiro, CP 68530, 21945-970, \\ Rio de Janeiro, RJ, Brazil \\ Correspondence should be addressed to Bruno Scardua, bruno.scardua@gmail.com \\ Received 22 May 2012; Accepted 22 July 2012 \\ Academic Editor: Kanishka Perera \\ Copyright ( $) 2012$ Bruno Scardua. This is an open access article distributed under the Creative \\ Commons Attribution License, which permits unrestricted use, distribution, and reproduction in \\ any medium, provided the original work is properly cited. \\ We prove that a transversely holomorphic foliation, which is transverse to the fibers of a fibration, \\ is a Seifert fibration if the set of compact leaves is not a zero measure subset. Similarly, we \\ prove that a finitely generated subgroup of holomorphic diffeomorphisms of a connected complex \\ manifold is finite provided that the set of periodic orbits is not a zero measure subset.
}

\section{Introduction}

Foliations transverse to fibrations are among the very first and simplest constructible examples of foliations, accompanied by a well-known transverse structure. These foliations are suspensions of groups of diffeomorphisms and their behavior is closely related to the action of the group in the fiber. For these reasons, many results holding for foliations in a more general context are first established for suspensions, that is, foliations transverse to a fibration. In this paper, we pursue this idea, but not restricted to it. We investigate versions of the classical stability theorems of Reeb [1,2], regarding the behavior of the foliation in a neighborhood of a compact leaf, replacing the finiteness of the holonomy group of the leaf by the existence of a sufficient number of compact leaves. This is done for transversely holomorphic (or transversely analytic) foliations.

Let $\eta=(E, \pi, B, F)$ be a (locally trivial) fibration with total space $E$, fiber $F$, base $B$, and projection $\pi: E \rightarrow B$. A foliation $F$ on $E$ is transverse to $\eta$ if: (1) for each $p \in E$, the leaf $L_{p}$ of $\mathcal{F}$ with $p \in L_{p}$ is transverse to the fiber $\pi^{-1}(q), q=\pi(p) ;(2) \operatorname{dim}(\mathcal{F})+\operatorname{dim}(F)=\operatorname{dim}(E) ;(3)$ for each leaf $L$ of $\mathcal{F}$, the restriction $\left.\pi\right|_{L}: L \rightarrow B$ is a covering map. A theorem of Ehresmann ([1] Chpter V) [2]) assures that if the fiber $F$ is compact, then conditions (1) and (2) together 
already imply (3). Such foliations are conjugate to suspensions and are characterized by their global holonomy ([1], Theorem 3, page 103 and [2], Theorem 6.1, page 59).

The codimension one case is studied in [3]. In [4], we study the case where the ambient manifold is a hyperbolic complex manifold. In [5], the authors prove a natural version of the stability theorem of Reeb for (transversely holomorphic) foliations transverse to fibrations. A foliation $\mathcal{F}$ on $M$ is called a Seifert fibration if all leaves are compact with finite holonomy groups.

The following stability theorem is proved in [5].

Theorem 1.1. Let $\mathcal{F}$ be a holomorphic foliation transverse to a fibration $\pi: E \stackrel{F}{\rightarrow} B$ with fiber $F$. If $\mathcal{F}$ has a compact leaf with finite holonomy group then $\mathcal{F}$ is a Seifert fibration.

It is also observed in [5] that the existence of a trivial holonomy compact leaf is assured if $\mathcal{F}$ is of codimension $k$ has a compact leaf, and the base $B$ satisfies $H^{1}(B, \mathbb{R})=$ $0, H^{1}(B, \mathrm{GL}(k, \mathbb{C}))=0$.

Since a foliation transverse to a fibration is conjugate to a suspension of a group of diffeomorphisms of the fiber, we can rely on the global holonomy of the foliation. As a general fact that holds also for smooth foliations, if the global holonomy group is finite then the foliation is a Seifert fibration. The proof of Theorem 1.1 relies on the local stability theorem of Reeb $[1,2]$ and the following remark derived from classical theorems of Burnside and Schur on finite exponent groups and periodic linear groups [5]: Let $G$ be a finitely generated subgroup of holomorphic diffeomorphisms of a connected complex manifold $F$. If each element of $G$ has finite order, then the subgroups with a common fixed point are finite.

We recall that a subset $X \subset M$ of a differentiable $m$-manifold has zero measure on $M$ if $M$ admits an open cover by coordinate charts $\varphi: U \subset M \rightarrow \varphi(U) \subset \mathbb{R}^{m}$ such that $\varphi(U \cap X)$ has zero measure with respect to the standard Lebesgue measure in $\mathbb{R}^{m}$. For sake of simplicity, if $X \subset \mathbb{R}^{n}$ is not a zero measure subset, then we will say that $X$ has positive measure and write $\mu(X)>0$. This may cause no confusion since, Indeed, we notice that if $X \subset M$ writes as a countable union $X=\bigcup_{n \in \mathbb{N}} X_{n}$ of subsets $X_{n} \subset M$ then $X$ has zero measure in $M$ if and only if $X_{n}$ has zero measure in $M$ for all $n \in \mathbb{N}$. In terms of our notation, we have therefore $\mu(X)>0$ if and only if $\mu\left(X_{n}\right)>0$ for some $n \in \mathbb{N}$.

In this paper, we improve Theorem 1.1 above by proving the following theorems.

Theorem 1.2. Let $\mp$ be a transversely holomorphic foliation transverse to a fibration $\pi: E \stackrel{F}{\rightarrow} B$ with fiber $F$ a connected complex manifold. Denote by $\Omega(\mathcal{F}) \subset E$ the union of all compact leaves of $\mathcal{F}$. Suppose that one have $\mu(\Omega(\mathcal{F}))>0$. Then $F$ is a Seifert fibration with finite global holonomy.

Parallel to this result we have the following version for groups.

Theorem 1.3. Let $G$ be a finitely generated subgroup of holomorphic diffeomorphisms of a complex connected manifold F. Denote by $\Omega(G)$ the subset of points $x \in F$ such that the $G$-orbit of $x$ is periodic. Assume that $\mu(\Omega(G))>0$. Then $G$ is a finite group.

As an immediate corollary of the above result, we get that, for a finitely generated subgroup $G \subset \operatorname{Diff}(F)$ of a complex connected manifold $F$, if the volume of the orbits gives an integrable function for some regular volume measure on $F$ then all orbits are periodic and the group is finite. This is related to results in [6]. 


\section{Holonomy and Global Holonomy}

Let $\mathcal{F}$ be a codimension $k$ transversely holomorphic foliation transverse to a fibration $\pi: E \stackrel{F}{\rightarrow}$ $B$ with fiber $F$, base $B$, and total space $E$. We always assume that $B, F$, and $E$ are connected manifolds. The manifold $F$ is a complex manifold.

\subsection{Holonomy}

For a given point $p \in E$, put $b=\pi(p) \in B$ and denote by $F_{b}=\pi^{1}(b) \subset E$ the fiber of $\pi$ over $b$, which is a complex biholomorphic to $F$. Given a point $p \in E$, we denote by $\operatorname{Hol}\left(\mathcal{F}, L_{p}\right)$ the holonomy group of the leaf $L_{p}$ through $p$ obtained by lifting to the leaves of $\mathcal{F}$, locally, closed paths in $L_{p}$ based on $p$, transversely to $F$ (see [1] for the construction of holonomy). Let us denote by $\operatorname{Diff}\left(F_{b}, p\right)$ the group of germs at $p$ of holomorphic diffeomorphisms of $F_{b}$ fixing $p \in$ $F_{b}$. The group $\operatorname{Diff}\left(F_{b}, p\right)$ is then identified with the group $\operatorname{Diff}\left(\mathbb{C}^{k}, 0\right)$ of germs at the origin $0 \in \mathbb{C}^{k}$ of complex diffeomorphisms, where $k=\operatorname{dim} F$.

This holonomy group $\operatorname{Hol}\left(\mathcal{F}, L_{p}\right)$ is formally defined as a conjugacy class of equivalence under diffeomorphism germs conjugation. Let us denote by $\operatorname{Hol}\left(L_{p}, F_{b}, p\right) \subset \operatorname{Diff}\left(F_{b}, p\right)$, its representative given by the local representation of this holonomy calculated with respect to the local transverse section induced by $F_{b}$ at the point $p \in F_{b}$. The group $\operatorname{Hol}\left(L_{p}, F_{b}, p\right)$ is therefore a subgroup of $\operatorname{Diff}\left(F_{b}, p\right)$ identified with a subgroup of $\operatorname{Diff}\left(\mathbb{C}^{k}, 0\right)$.

\subsection{Global Holonomy}

As it is well known, the fundamental group $\pi_{1}(B)$ acts on the group of holomorphic diffeomorphisms of the manifold $F \operatorname{Diff}(F)$, by what we call the global holonomy representation. This consists of a group homomorphism $\varphi: \pi_{1}(B, b) \rightarrow \operatorname{Diff}(F)$, obtained by lifting closed paths in $B$ to the leaves of $\mathcal{F}$ via the covering maps $\left.\pi\right|_{L}: L \rightarrow B$, where $L$ is a leaf of $\mathcal{F}$. The image of this representation is the global holonomy $\operatorname{Hol}(\mathcal{F})$ of $\mathcal{F}$, and its construction shows that $\mathcal{F}$ is conjugated to the suspension of its global holonomy ([1], Theorem 3, page 103). Given a base point $b \in B$, we will denote by $\operatorname{Hol}\left(\mathcal{F}, F_{b}\right) \subset \operatorname{Diff}\left(F_{b}\right)$ the representation of the global holonomy of $\mathcal{F}$ based at $b$.

From the classical theory [1], chapter V and [5] we have the following.

Proposition 2.1. Let $\mp$ be a foliation on $E$ transverse to the fibration $\pi: E \rightarrow B$ with fiber F. Fix a point $p \in E, b=\pi(p)$ and denote by $L$ the leaf that contains $p$.

(1) The holonomy group $\operatorname{Hol}\left(L, F_{b}, p\right)$ of $L$ is the subgroup of the global holonomy $\operatorname{Hol}\left(\mathcal{F}, F_{b}\right) \subset \operatorname{Diff}\left(F_{b}\right)$ of those elements that have $p$ as a fixed point.

(2) Given another intersection point $q \in L \cap F_{b}$, there is a global holonomy map $h \in \operatorname{Hol}\left(\mp, F_{b}\right)$ such that $h(p)=q$.

(3) Suppose that the global holonomy Hol (F) is finite. If $\mp$ has a compact leaf then it is a Seifert fibration, that is, all leaves are compact with finite holonomy group.

(4) If $\mathcal{F}$ has a compact leaf $L_{0} \in \mathcal{F}$ then each point $p \in F_{b} \cap L_{0}$ has periodic orbit in the global holonomy $\operatorname{Hol}(\mathcal{F})$. In particular, there are $\ell \in \mathbb{N}$ and $p \in F$ such that $h^{\ell}(p)=p$ for every $h \in \operatorname{Hol}(\mp)$. 


\section{Periodic Groups and Groups of Finite Exponent}

First we recall some facts from the theory of Linear groups. Let $G$ be a group with identity $e_{G} \in$ $G$. The group is periodic if each element of $G$ has finite order. A periodic group $G$ is periodic of bounded exponent if there is an uniform upper bound for the orders of its elements. This is equivalent to the existence of $m \in \mathbb{N}$ with $g^{m}=1$ for all $g \in G$ (cf. [5]). Because of this, a group which is periodic of bounded exponent is also called a group of finite exponent. Given $R$ a ring with identity, we say that a group $G$ is $R$-linear if it is isomorphic to a subgroup of the matrix group $\mathrm{GL}(n, R)$ (of $n \times n$ invertible matrices with coefficients belonging to $R$ ) for some $n \in \mathbb{N}$. We will consider complex linear groups. The following classical results are due to Burnside and Schur.

Theorem 3.1. With respect to complex linear groups one has the following.

(1) Burnside, [7] A (not necessarily finitely generated) complex linear group $G \subset \mathrm{GL}(k, \mathbb{C})$ of finite exponent $\ell$ has finite order; actually we have $|G| \leq \ell^{k^{2}}$.

(2) Schur, [8] Every finitely generated periodic subgroup of $\mathrm{GL}(n, \mathbb{C})$ is finite.

Using these results, we obtain in [5].

Lemma 3.2 (see Lemmas 2.3, 3.2, and 3.3 [5]). About periodic groups of germs of complex diffeomorphisms one has the following.

(1) A finitely generated periodic subgroup $G \subset \operatorname{Diff}\left(\mathbb{C}^{k}, 0\right)$ is necessarily finite.

(2) A (not necessarily finitely generated) subgroup $G \subset \operatorname{Diff}\left(\mathbb{C}^{k}, 0\right)$ of finite exponent is necessarily finite.

(3) Let $G \subset \operatorname{Diff}\left(\mathbb{C}^{k}, 0\right)$ be a finitely generated subgroup. Assume that there is an invariant connected neighborhood $W$ of the origin in $\mathbb{C}^{k}$ such that each point $x$ is periodic for each element $g \in G$. Then $G$ is a finite group.

(4) Let $G \subset \operatorname{Diff}\left(\mathbb{C}^{k}, 0\right)$ be a (not necessarily finitely generated) subgroup such that for each point $x$ close enough to the origin, the pseudoorbit of $x$ is finite of (uniformly bounded) order $\leq \ell$ for some $\ell \in \mathbb{N}$, then $G$ is finite.

Given a subgroup $G \subset \operatorname{Diff}(F)$ and a point $p \in F$ the stabilizer of $p$ in $G$ is the subgroup $G(p) \subset G$ of the elements $f \in G$ such that $f(p)=p$. From the above one has the following.

Proposition 3.3. Let $G \subset \operatorname{Diff}(F)$ be a (not necessarily finitely generated) subgroup of holomorphic diffeomorphisms of a connected complex manifold $F$.

(1) If $G$ is periodic and finitely generated or $G$ is periodic of finite exponent, then each stabilizer subgroup of $G$ is finite.

(2) Assume that there is a point $p \in F$ which is fixed by $G$ and a fundamental system of neighborhoods $\left\{U_{v}\right\}_{v}$ of $p$ in $F$ such that each $U_{v}$ is invariant by $G$, the orbits of $G$ in $U_{v}$ are periodic (not necessarily with uniformly bounded orders). Then $G$ is a finite group.

(3) Assume that $G$ has a periodic orbit $\left\{x_{1}, \ldots, x_{r}\right\} \subset F$ such that for each $j \in\{1, \ldots, r\}$, there is a fundamental system of neighborhoods $U_{v}^{j}$ of $x_{j}$ with the property that $U_{v}=\bigcup_{j=1}^{r} U_{v}^{j}$ is invariant under the action of $G, U_{v}^{j} \cap U_{v}^{\ell}=\emptyset$ if $j \neq \ell$, and each orbit in $U_{v}$ is periodic. Then $G$ is periodic. 
Proof. In order to prove (1), we consider the case where $G$ has a fixed point $p \in F$. We identify the group $\mathcal{G}_{p} \subset \operatorname{Diff}(F, p)$, of germs at $p \in F$ of maps in $G \subset \operatorname{Diff}(F)$, with a subgroup of $\operatorname{Diff}\left(\mathbb{C}^{n}, 0\right)$ where $n=\operatorname{dim} F$. If $G$ is finitely generated and periodic, the group $\mathcal{G}_{p}$ is finitely generated and periodic. By Lemma $3.2(1)$, the group $\mathcal{G}_{p}$ is finite and by the Identity principle the group $G$ is also finite of same order. If $G$ is periodic of finite exponent then the group $\mathcal{G}_{p}$ is periodic of finite exponent. By Lemma $3.2(2)$, the group $\mathcal{G}_{p}$ is finite and by the Identity principle the group $G$ is also finite of same order. This proves (1).

As for (2), since $U_{v}$ is $G$-invariant, each element $g \in G$ induces by restriction to $U_{v}$ an element of a group $G_{v} \subset \operatorname{Diff}\left(U_{v}\right)$. It is observed in [5] (proof of Lemma 3.5) that the finiteness of the orbits in $U_{v}$ implies that $G_{v}$ is periodic. By the Identity principle, the group $G$ is also periodic of the same order. Since $G=G(p)$, (2) follows from (1). (3) is proved like the first part of (2).

The following simple remark gives the finiteness of finite exponent groups of holomorphic diffeomorphisms having a periodic orbit.

Proposition 3.4 (Finiteness lemma). Let $G$ be a subgroup of holomorphic diffeomorphisms of a connected complex manifold $F$. Assume that

(1) $G$ is periodic of finite exponent or $G$ is finitely generated and periodic,

(2) G has a finite orbit in F.

Then $G$ is finite.

Proof. Fix a point $x \in F$ with finite orbit, we can write $\mathcal{O}_{G}(x)=\left\{x_{1}, \ldots, x_{k}\right\}$ with $x_{i} \neq x_{j}$ if $i \neq j$. Given any diffeomorphism $f \in G$, we have $\mathcal{O}_{G}(f(x))=\mathcal{O}_{G}(x)$ so that there exists an unique element $\sigma \in S_{k}$ of the symmetric group such that $f\left(x_{j}\right)=x_{\sigma_{f}(j)}$, for all $j=1, \ldots, k$. We can therefore define a map

$$
\eta: G \rightarrow S_{k}, \quad \eta(f)=\sigma_{f}
$$

Now, if $f, g \in G$ are such that $\eta(f)=\eta(g)$, then $f\left(x_{j}\right)=g\left(x_{j}\right)$, for all $j$ and therefore $h=$ $f g^{-1} \in G$ fixes the points $x_{1}, \ldots, x_{k}$. In particular, $h$ belongs to the stabilizer $G_{x}$. By Proposition 3.3(1) and (2) (according to $G$ is finitely generated or not), the group $G_{x}$ is finite. Thus, the map $\eta: G \rightarrow S_{k}$ is a finite map. Since $S_{k}$ is a finite group, this implies that $G$ is finite as well.

\section{Measure and Finiteness}

The following lemma paves the way to Theorems 1.2 and 1.3.

Lemma 4.1. Let $G$ be a subgroup of complex diffeomorphisms of a connected complex manifold $F$. Denote by $\Omega(G)$ the set of points $x \in F$ such that the orbit $\mathcal{O}_{G}(x)$ is periodic. If $\mu(\Omega(G))>0$ then $G$ is a periodic group of finite exponent.

Proof. We have $\Omega(G)=\left\{x \in F: \# \mathcal{O}_{G}(x)<\infty\right\}=\bigcup_{k=1}^{\infty}\left\{x \in F: \# \mathcal{O}_{G}(x) \leq k\right\}$, therefore there is some $k \in \mathbb{N}$ such that

$$
\mu\left(\left\{x \in F: \# \mathcal{O}_{G}(x) \leq k\right\}\right)>0 .
$$


In particular, given any diffeomorphism $f \in G$ we have

$$
\mu\left(\left\{x \in F: \# \mathcal{O}_{f}(x) \leq k\right\}\right)>0 .
$$

In particular, there is $k_{f} \leq k$ such that the set $X=\left\{x \in F: f^{k_{f}}(x)=x\right\}$ has positive measure. Since $X \subset F$ is an analytic subset, this implies that $X=F$ (a proper analytic subset of a connected complex manifold has zero measure). Therefore, we have $f^{k_{f}}=\operatorname{Id}$ in $F$. This shows that $G$ is periodic of finite exponent.

Proof of Theorem 1.2. Fix a base point $b \in B$. By Proposition 2.1, the compact leaves correspond to periodic orbits of the global holonomy $\operatorname{Hol}\left(\mathcal{F}, F_{b}\right)$. Therefore, by the hypothesis the global holonomy $G=\operatorname{Hol}\left(\mathcal{F}, F_{b}\right)$ satisfies the hypothesis of Lemma 4.1. By this lemma, the global holonomy is periodic of finite exponent. Since this group has some periodic orbit, by the Finiteness lemma (Proposition 3.4) the global holonomy group is finite. By Proposition 2.1(3), the foliation is a Seifert fibration.

\section{2 .}

The construction of the suspension of a group action gives Theorem 1.3 from Theorem

Proof of Theorem 1.3. Since $G$ is finitely generated, there are a compact connected manifold $B$ and a representation $\varphi: \pi_{1}(B) \rightarrow \operatorname{Diff}(F)$ such that the image $\varphi\left(\pi_{1}(B)\right)=G$. The manifold $B$ is not necessarily a complex manifold, but this makes no difference in our argumentation based only on the fact that the foliation is transversely holomorphic. Denote by $\mathcal{F}$ the suspension foliation of the fibre bundle $\pi: E \rightarrow B$ with fiber $F$ which has global holonomy conjugate to $G$. The periodic orbits of $G$ in $F$ correspond in a natural way to the leaves of $\mathscr{F}$ which have finite order with respect to the fibration $\pi: E \rightarrow B$, that is, the leaves which intersect the fibers of $\pi: E \rightarrow B$ only at a finite number of points. Thus, because the basis is compact, each such leaf (corresponding to a finite orbit of $G$ ) is compact. By the hypothesis, we have $\mu(\Omega(\mathscr{F}))>0$. By Theorem 1.2 the global holonomy $\operatorname{Hol}(\mathscr{F})$ is finite. Thus, the group $G$ is finite.

\section{References}

[1] C. Camacho and A. Lins Neto, Geometric Theory of Foliations, Birkhäuser, Boston, Mass, USA, 1985.

[2] C. Godbillon, "Foliations," in Geometric Studies, Progress in Mathematics, 98, Birkhäuser, Basel, Switzerland, 1991.

[3] B. Scárdua, "On complex codimension-one foliations transverse fibrations," Journal of Dynamical and Control Systems, vol. 11, no. 4, pp. 575-603, 2005.

[4] B. A. Scárdua, "Holomorphic foliations transverse to fibrations on hyperbolic manifolds," Complex Variables. Theory and Application, vol. 46, no. 3, pp. 219-240, 2001.

[5] F. Santos and B. Scardua, "Stability of complex foliations transverse to fibrations," Proceedings of the American Mathematical Society, vol. 140, no. 9, pp. 3083-3090, 2012.

[6] B. Scárdua, "Complex vector fields having orbits with bounded geometry," The Tohoku Mathematical Journal, vol. 54, no. 3, pp. 367-392, 2002.

[7] W. Burnside, "On criteria for the finiteness of the order of a group of linear substitutions," Proceedings of the London Mathematical Society, vol. 3, no. 2, pp. 435-440.

[8] I. Schur, "Über Gruppen periodischer substitutionen," Sitzungsber. Preuss. Akad. Wiss, pp. 619-627, 1911. 


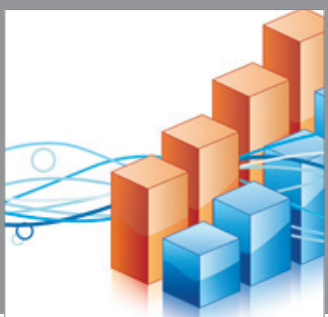

Advances in

Operations Research

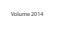

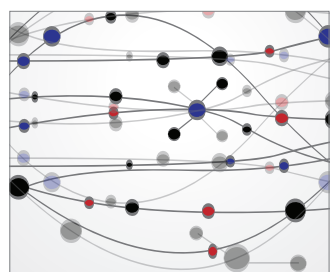

\section{The Scientific} World Journal
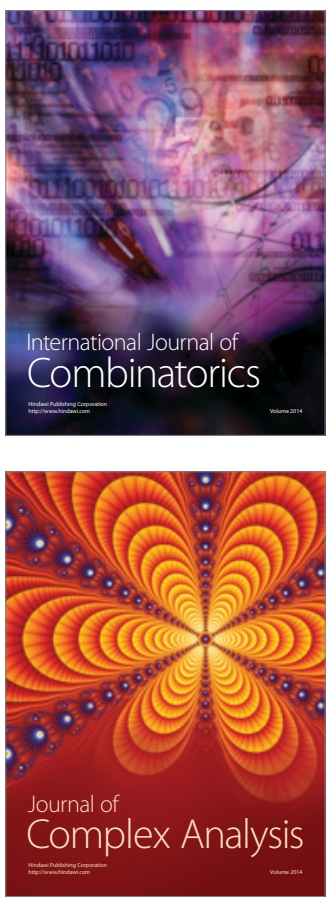

International Journal of

Mathematics and

Mathematical

Sciences
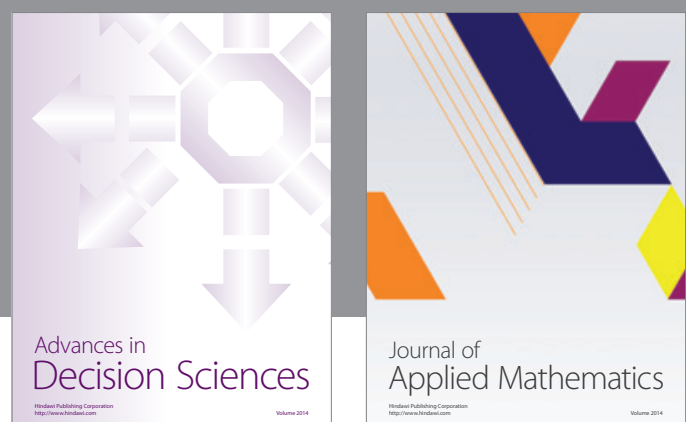

Journal of

Applied Mathematics
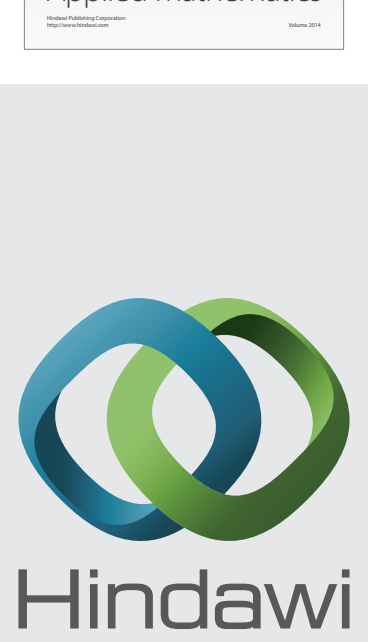

Submit your manuscripts at http://www.hindawi.com
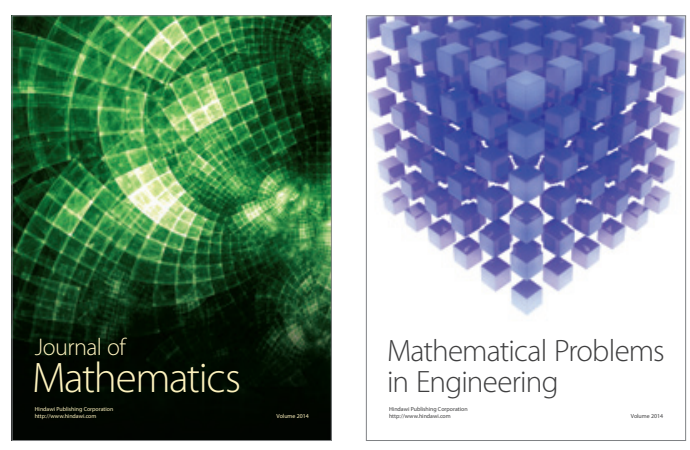

Mathematical Problems in Engineering
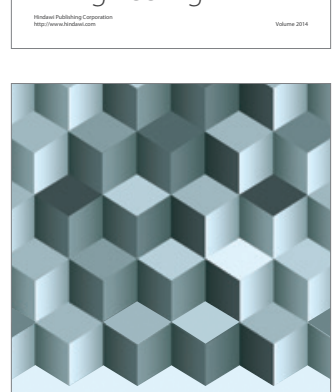

Journal of

Function Spaces
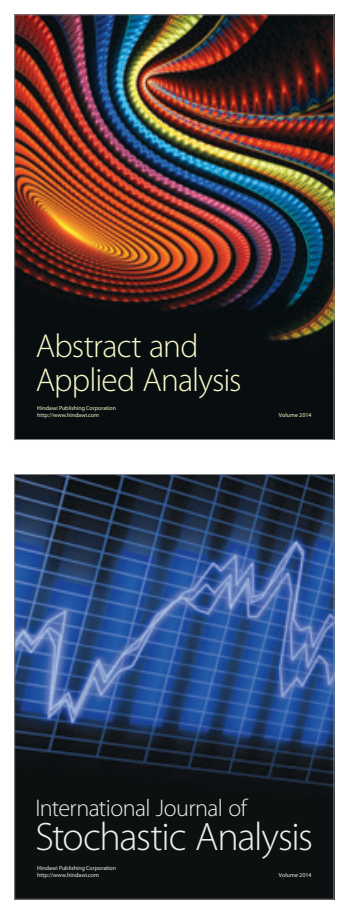

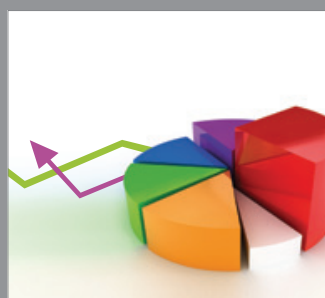

ournal of

Probability and Statistics

Promensencen
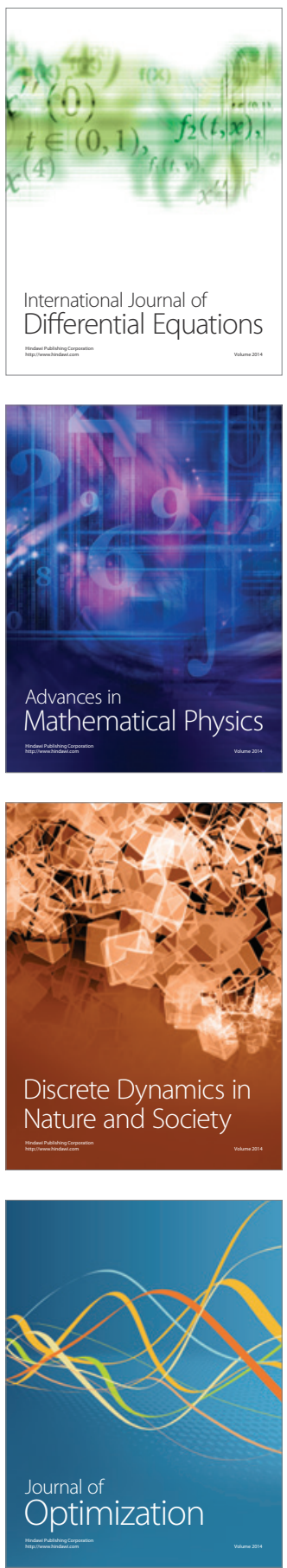